\title{
Galactic Pal-eontology: abundance analysis of the disrupting globular cluster Palomar $5^{\star}$
}

\author{
Andreas Koch ${ }^{1}$ and Patrick Côté ${ }^{2}$ \\ 1 Department of Physics, Lancaster University, Lancaster LA1 4YB, UK \\ e-mail: a.koch1@lancaster.ac.uk \\ 2 National Research Council of Canada, Herzberg Astronomy and Astrophysics Program, 5071 West Saanich Road, Victoria, \\ BC V9E 2E7, Canada
}

Received 10 October 2016 / Accepted 21 February 2017

\begin{abstract}
We present a chemical abundance analysis of the tidally disrupted globular cluster (GC) Palomar 5. By co-adding high-resolution spectra of 15 member stars from the cluster's main body, taken at low signal-to-noise with the Keck/HIRES spectrograph, we were able to measure integrated abundance ratios of 24 species of 20 elements including all major nucleosynthetic channels (namely the light element $\mathrm{Na}$; $\alpha$-elements $\mathrm{Mg}, \mathrm{Si}, \mathrm{Ca}$, Ti; Fe-peak and heavy elements $\mathrm{Sc}, \mathrm{V}, \mathrm{Cr}, \mathrm{Mn}, \mathrm{Co}, \mathrm{Ni}, \mathrm{Cu}, \mathrm{Zn}$; and the neutron-capture elements $\mathrm{Y}, \mathrm{Zr}, \mathrm{Ba}, \mathrm{La}, \mathrm{Nd}, \mathrm{Sm}, \mathrm{Eu}$ ). The mean metallicity of $-1.56 \pm 0.02 \pm 0.06 \mathrm{dex}$ (statistical and systematic errors) agrees well with the values from individual, low-resolution measurements of individual stars, but it is lower than previous high-resolution results of a small number of stars in the literature. Comparison with Galactic halo stars and other disrupted and unperturbed GCs renders Pal 5 a typical representative of the Milky Way halo population, as has been noted before, emphasizing that the early chemical evolution of such clusters is decoupled from their later dynamical history. We also performed a test as to the detectability of light element variations in this co-added abundance analysis technique and found that this approach is not sensitive even in the presence of a broad range in sodium of $\sim 0.6 \mathrm{dex}$, a value typically found in the old halo GCs. Thus, while methods of determining the global abundance patterns of such objects are well suited to study their overall enrichment histories, chemical distinctions of their multiple stellar populations is still best obtained from measurements of individual stars.
\end{abstract}

Key words. techniques: spectroscopic - stars: abundances - Galaxy: abundances - Galaxy: evolution - Galaxy: halo globular clusters: individual: Palomar 5

\section{Introduction}

Globular clusters (GCs) are amongst the oldest objects in the Universe and offer unique testbeds to probe internal evolutionary processes at those early times, as well as the build-up of the Galactic halo. As to the first point, it is now well-established that any GC observed to date hosts several stellar populations distinct in age, their light-element abundances (e.g., Piotto et al. 2007; Gratton et al. 2012; Milone et al. 2017), and, in a few cases, in some heavy elements (e.g., Marino et al. 2011; Roederer et al. 2016). In the latter case, those GCs host a fraction of stars usually differing also in their iron content, and in each of the metallicity subgroups there is evidence of variations in the light elements (e.g., Carretta et al. 2010; Marino et al. 2011). These light-element variations point to proton-capture reactions acting in the hot interiors of stars associated with the first generations to have formed within the clusters. Even in observations with lownumber statistics that do not allow for a representative sampling of the omnipresent Na-O anti-correlation (Carretta et al. 2009), abundance anomalies can be found such as spreads in heavy elements, that indicate complex enrichment histories (e.g., Koch \& McWilliam 2014; Hanke et al. 2017).

Secondly, comparisons of the global chemical element abundances of GCs with those of the stellar Galactic halo have

\footnotetext{
$\star$ Full Table 3 is is only available at the CDS via anonymous ftp to cdsarc.u-strasbg. fr (130.79.128.5) or via

http://cdsarc.u-strasbg.fr/viz-bin/qcat?]/A+A/601/A41
}

implications for the accretion history of the Milky Way. In this regard, several candidates have been chemically and kinematically identified as having originated in the disrupted Sagittarius dwarf galaxy (e.g., Law \& Majewski 2010; Sbordone et al. 2015). Moreover, by studying light-element (in particular $\mathrm{CN}$ and $\mathrm{CH}$ ) variations in $\mathrm{GC}$ and halo stars, the fraction of the halo that was donated by disrupted satellites can be efficiently constrained (e.g., Martell \& Grebel 2010).

Palomar 5 (hereafter Pal 5) is one of the most prominent examples of a stellar system in severe tidal disruption, having lost up to $90 \%$ of its mass into tidal tails that stretch over $10^{\circ}$ across the sky (Odenkirchen et al. 2002, 2003). Previous photometric (e.g., Sandage \& Hartwick 1977; Dotter et al. 2011) and low-resolution spectroscopic studies have identified it as a moderately metal-poor $([\mathrm{Fe} / \mathrm{H}] \sim-1.4 \mathrm{dex})$ system (Smith 1985; Kuzma et al. 2015; Ishigaki et al. 2015). The latter two studies extended their analysis over broad parts of the tidal streams and they recovered a strong radial velocity gradient that is in line with simulations of the tidal disruption and can serve as input to improving halo mass models (Dehnen et al. 2004; Odenkirchen et al. 2009; Pearson et al. 2015). However, no metallicity gradient across the tails has been found. Smith et al. (2002) performed the first and only measurements of four stars at high spectral resolution and high signal-to-noise ratio (SNR), suggesting that Pal 5 resembles the Galactic halo and higher-mass GCs in many regards; one exception was a lower-than-average $[\alpha / \mathrm{Fe}]$ abundance ratio, which, at $\sim 0.16 \mathrm{dex}$, was found to lie 
significantly below the plateau value of $\sim 0.4$ dex inhabited by GC and field stars at similar metallicities. Furthermore, Smith (1985) and Smith et al. (2002) detected large abundance variations in the light elements $(\mathrm{C}, \mathrm{N}, \mathrm{Na}$, and $\mathrm{Al})$ and concluded that whatever evolutionary processes are responsible for those variations in strongly disrupted clusters are oblivious to the GC's present-day mass and that they were already imprinted at early times when the clusters still retained their initial mass. Conversely, Carretta et al. (2010) found that the extent of the variations in light elements due to proton-capture processing is tightly related to the present-day total mass of clusters, which is the main driving parameter.

Many remote Milky Way GCs are faint systems, which leads to time-expensive observation strategies; thus one path to obtaining detailed chemical abundance information is to use integrated light spectroscopy (e.g., McWilliam \& Bernstein 2008) or to coadd individual spectra at low SNR to emulate a higher-quality spectrum of a known underlying stellar population (Koch et al. 2009; Koch \& Côté 2010). Following our work on the outer halo clusters $\mathrm{Pal} 3$ and $\mathrm{Pal} 4$, we now turn to the closer $\left(R_{\odot}=23 \mathrm{kpc}\right)$ object Pal 5.

In Sect. 2 we describe our target list and observations, followed by a discussion of the radial velocity measurements and cluster membership assessments in Sect. 3. Details on the coadded abundance determination and error analysis are given in Sect. 4 and we present the results in Sect. 5, with a focus on comparison with other GCs in Sect. 6. In Sect. 7 we briefly comment on the detectability of abundance variations with our approach, before concluding in Sect. 8.

\section{Targets and observations}

The Pal 5 data discussed here were taken as part of a broader program to study the internal dynamics of outer halo GCs (see, e.g., Côté et al. 2002; Jordi et al. 2009; Baumgardt et al. 2009; Frank et al. 2012). Our Pal 5 target stars were chosen from the red giant branch (RGB) and asymptotic giant branch (AGB) sequences identified in the early photometric studies of Sandage \& Hartwick (1977, SH77) and the unpublished photometry and astrometry from Cudworth, Schweitzer, and Majewski (CSM; see Schweitzer et al. 1993). Our target stars reach out to $\sim 2$ half-light radii and all lie well within the cluster's tidal radius, avoiding the cluster's tidal features (Odenkirchen et al. 2003). Of course, the concept of tidal radius for such a highly disturbed object is largely meaningless and we refrain from investigating possible spatial trends in kinematics or abundance. Properties of the target stars and HIRES observations are given in Table 1. In Fig. 1, we show their location in a color-magnitude diagram (CMD) based on photometry from the Sloan Digital Sky Survey (SDSS; Alam et al. 2015).

\section{Radial velocities and membership}

Our observations were taken on May 31, 1998 using the HIRES echelle spectrograph (Vogt et al. 1994) on the Keck I telescope with the $\mathrm{C} 1$ decker (slit width of $0.86^{\prime \prime}$ ) and $1 \times 2$ binning, which provides a spectral resolution of $R=45000$ and a full spectra coverage of $4300-6720 \AA$. The data were reduced with the Makee $^{1}$ pipeline. The radial velocity of each star was measured

\footnotetext{
1 MAKEE was developed by T. A. Barlow specifically for reduction of Keck HIRES data. It is freely available on the World Wide Web at the Keck Observatory home page, http://www2 . keck . hawaii . edu/ inst/hires/makeewww
}

Table 1. Log of target stars.

\begin{tabular}{ccccc}
\hline \hline \multicolumn{1}{c}{ Star $^{a}$} & $\begin{array}{c}\alpha \\
(\mathrm{J} 2000.0)\end{array}$ & $\begin{array}{c}\delta \\
(\mathrm{J} 2000.0)\end{array}$ & $\begin{array}{c}\text { Exp. time } \\
{[\mathrm{s}]}\end{array}$ & $\begin{array}{c}S / N^{b} \\
{\left[\text { pixel }^{-1}\right]}\end{array}$ \\
\hline CSM-003 & 151615.93 & -000928.79 & 180 & 6 \\
CSM-029 & 151613.99 & -000933.39 & 300 & 7 \\
CSM-030 & 151616.37 & -001029.76 & 300 & 6 \\
CSM-032 & 151609.52 & -000239.64 & 240 & 7 \\
CSM-045 & 151620.85 & -000842.72 & 180 & 9 \\
CSM-174 & 151607.67 & -001018.64 & 180 & 7 \\
CSM-598 & 151619.18 & -001131.11 & 600 & 8 \\
SH77-E & 151558.83 & -000517.02 & 180 & 10 \\
SH77-F & 151556.05 & -000605.67 & 180 & 12 \\
SH77-G & 151608.61 & -000803.19 & 180 & 8 \\
SH77-H & 151552.54 & -000740.47 & 180 & 7 \\
SH77-J & 151549.64 & -000700.73 & 240 & 7 \\
SH77-K & 151606.47 & -000700.91 & 240 & 7 \\
SH77-L & 151601.95 & -000802.75 & 240 & 8 \\
SH77-M & 151546.08 & -000908.42 & 240 & 6 \\
SH77-N & 151559.45 & -000859.69 & 240 & 6 \\
SH77-14 & 151608.26 & -000738.01 & 600 & 7 \\
SH77-U & 151554.73 & -000654.95 & 300 & 5 \\
\hline
\end{tabular}

Notes. ${ }^{(a)}$ Star-IDs from SH77 and CSM. ${ }^{(b)}$ Given at $6600 \AA$.

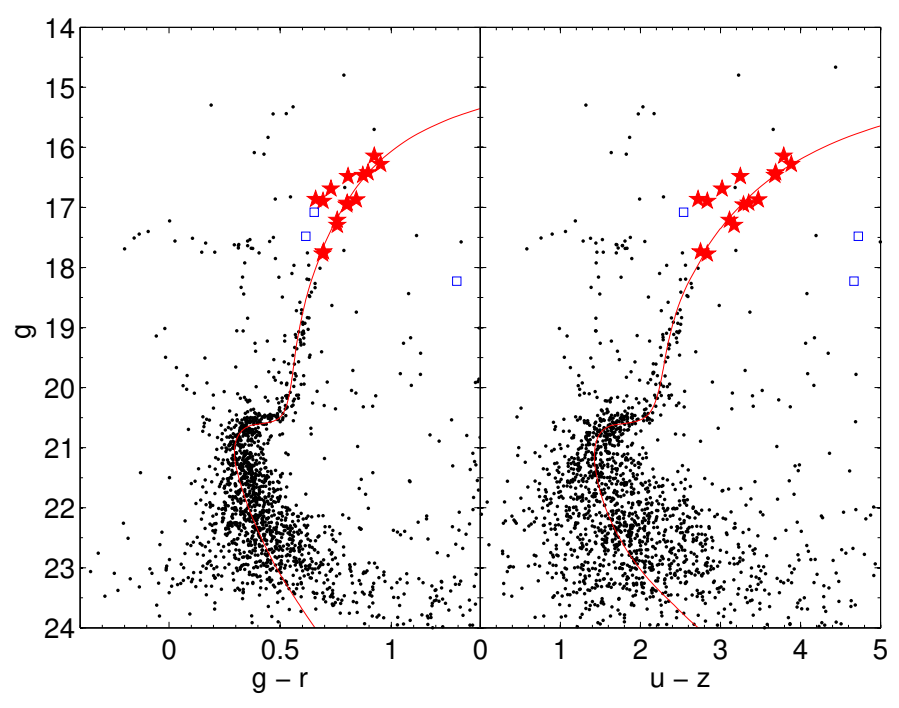

Fig. 1. CMD using SDSS photometry of stars within two half-light radii. Blue squares indicate foreground stars, open red symbols are AGB candidates, while our RGB targets are shown as solid stars. Dartmouth isochrones of the GC's parameters are shown as red lines.

by cross-correlating its spectrum against that of a master template created during each run (see below) from observations of the International Astronomical Union's (IAU) standard stars. In order to minimize possible systematic effects, a master template for each observing run was derived from an identical subsample of IAU standard stars. From each cross-correlation function, we measured both $v_{\mathrm{HC}}$, the heliocentric radial velocity, and $R_{\mathrm{TD}}$, the Tonry \& Davis (1979) estimator of the strength of the crosscorrelation peak.

During our broader Keck program, which spanned seven observing runs in 1998 and 1999 (13 nights in total), we obtained 53 distinct radial velocity measurements for 23 different RGB 
Table 2. Properties of the target stars.

\begin{tabular}{cccccccccccc}
\hline \hline Star & $u$ & $g$ & $r$ & $i$ & $z$ & $v_{\mathrm{HC}}\left[\mathrm{km} \mathrm{s}^{-1}\right]$ & Type $^{a}$ & $T_{\text {eff }}[\mathrm{K}]$ & $\log g$ & $\xi\left[\mathrm{km} \mathrm{s}^{-1}\right]$ & {$[\mathrm{Fe} / \mathrm{H}]_{\mathrm{MgT}}$} \\
\hline CSM-003 & 18.934 & 16.869 & 16.027 & 15.673 & 15.461 & $-52.19 \pm 0.50$ & RGB & 4473 & 1.40 & 1.76 & -1.32 \\
CSM-029 & 19.205 & 17.296 & 16.538 & 16.217 & 16.033 & $-57.42 \pm 0.54$ & RGB & 4607 & 1.67 & 1.68 & -1.33 \\
CSM-045 & 18.564 & 16.279 & 15.327 & 14.921 & 14.679 & $-59.28 \pm 0.37$ & RGB & 4317 & 1.03 & 1.86 & -1.45 \\
SH77-F & 18.622 & 16.419 & 15.524 & 15.157 & 14.933 & $-58.51 \pm 0.40$ & RGB & 4410 & 1.16 & 1.80 & -1.43 \\
SH77-G & 18.681 & 16.464 & 15.592 & 15.217 & 15.000 & $-58.28 \pm 0.45$ & RGB & 4418 & 1.19 & 1.80 & -1.42 \\
SH77-K & 18.950 & 16.926 & 16.125 & 15.783 & 15.597 & $-59.25 \pm 0.46$ & RGB & 4531 & 1.46 & 1.73 & -1.33 \\
SH77-L & 18.905 & 16.955 & 16.155 & 15.816 & 15.616 & $-58.90 \pm 0.47$ & RGB & 4536 & 1.48 & 1.72 & -1.38 \\
SH77-N & 19.057 & 17.210 & 16.453 & 16.128 & 15.943 & $-60.32 \pm 0.59$ & RGB & 4601 & 1.63 & 1.68 & -1.33 \\
SH77-14 & 19.437 & 17.772 & 17.082 & 16.787 & 16.601 & $-58.41 \pm 0.53$ & RGB & 4731 & 1.94 & 1.60 & -1.32 \\
SH77-U & 19.329 & 17.735 & 17.040 & 16.744 & 16.578 & $-59.09 \pm 0.90$ & RGB & 4722 & 1.92 & 1.61 & -1.13 \\
\hline CSM-032 & 18.585 & 16.894 & 16.201 & 15.911 & 15.749 & $-59.00 \pm 0.58$ & AGB & 4737 & 1.47 & 1.60 & -1.44 \\
CSM-174 & 18.410 & 16.481 & 15.676 & 15.344 & 15.161 & $-61.14 \pm 0.50$ & AGB & 4545 & 1.17 & 1.72 & -1.49 \\
SH77-E & 18.393 & 16.142 & 15.219 & 14.830 & 14.601 & $-57.48 \pm 0.38$ & AGB & 4358 & 0.88 & 1.83 & -1.46 \\
SH77-H & 18.514 & 16.690 & 15.961 & 15.648 & 15.493 & $-57.24 \pm 0.58$ & AGB & 4653 & 1.33 & 1.65 & -1.37 \\
SH77-J & 18.466 & 16.863 & 16.203 & 15.911 & 15.744 & $-57.38 \pm 0.62$ & AGB & 4773 & 1.48 & 1.57 & -1.58 \\
\hline CSM-030 & 21.280 & 17.481 & 16.865 & 16.533 & 16.558 & $-38.32 \pm 0.57$ & FG & - & - & - & - \\
CSM-598 & 20.772 & 18.226 & 16.932 & 16.409 & 16.104 & $-14.33 \pm 0.61$ & FG & - & - & - & - \\
SH77-M & 18.500 & 17.079 & 16.426 & 16.131 & 15.959 & $20.18 \pm 1.11$ & FG & - & - & - & - \\
\hline
\end{tabular}

Notes. ${ }^{(a)}$ Stellar type based on CMD and spectral properties: red giant branch (RGB), asymptotic giant branch (AGB), or foreground (FG) dwarf.

and subgiant stars belonging to Pal 3, Pal 4, Pal 5, Pal 14, NGC 7492, and NGC 2419. Using these repeat measurements and following the procedures described in Vogt et al. (1995), we derived an empirical relationship between our radial velocity uncertainties, $\epsilon\left(v_{\mathrm{HC}}\right)$, and the strength of cross-correlation peak: $\epsilon\left(v_{\mathrm{HC}}\right)=\alpha /\left(1+R_{\mathrm{TD}}\right)$, where $\alpha=9.0_{-1.6}^{+2.4} \mathrm{~km} \mathrm{~s}^{-1}(90 \%$ confidence limits). The final radial velocities and their uncertainties are recorded in Table 2.

Several spectroscopic studies have since been carried out for Pal 5. All of the four stars analyzed by Smith et al. (2002), using Keck/HIRES at high SNR but lower resolution $\left(R=34000^{2}\right)$ than in the present work, are included in our data set as well, whereas none of the stars are included in Odenkirchen's et al. (2009) kinematic study. Odenkirchen et al. (2002) performed a kinematic study on their spectra obtained with the Ultraviolet and Visual Echelle Spectrograph (UVES) at high-resolution, but low SNR $\left(\sim 10 \mathrm{pixel}^{-1}\right)$, of which ten stars are also in our present sample. We observed 11 stars in common with the work of Kuzma et al. (2015), who obtained velocity and calcium-triplet metallicity measurements based on low-resolution $(R \sim 10000)$ spectroscopy with the AAOmega multi-fibre instrument. Finally, one of our stars is also included in the data of Ishigaki et al. (2016) who used low-resolution $(R \sim 7000)$ spectra to gather kinematics, metallicities, and $[\alpha / \mathrm{Fe}]$ ratios.

The mean heliocentric velocity of $-58.3 \pm 0.5 \mathrm{~km} \mathrm{~s}^{-1}$ we find for Pal 5 is in excellent agreement with all previous studies of the central regions to within the uncertainties. The dispersion of $1.8 \pm 0.3 \mathrm{~km} \mathrm{~s}^{-1}$ from our data is slightly higher than the literature values of typically $1.1 \pm 0.3 \mathrm{~km} \mathrm{~s}^{-1}$, but this may be due to the lower number of stars we observed or to our confinement to the more central parts of the cluster. Three of our targets turned out to be non-members based on their strongly deviating velocities. While the presence of a strong velocity gradient throughout this tidal system (e.g., Odenkirchen et al. 2002) could in

\footnotetext{
2 This is the same value as used in our previous study of Pal 4; Koch \& Côté (2010).
}

principle render these stars related to Pal 5, the appearance of their spectral features such as broad, gravity-sensitive Mg triplet lines or the Ca $6162 \AA$-line, clearly indicates that they are foreground dwarfs. As the CMD in Fig. 1 implies, five of the targets are located on the AGB, as was also suggested for four of them by Odenkirchen et al. (2002). The separation of our sample into $\mathrm{RGB}, \mathrm{AGB}$, and foreground stars is indicated in Table 2.

\section{Analysis}

The original aim in acquiring this data set was to study the kinematics of Galactic halo clusters (see Sect. 2) and so the exposure times were chosen to be in the range of three-ten minutes, thus operating at low SNR (Table 1; see also Odenkirchen et al. 2002). While this SNR is adequate for measuring accurate radial velocities, a detailed chemical analysis of individual stars is precluded. Thus, we resort to our method of co-adding spectra in the manner outlined in Koch et al. (2009) and Koch \& Côté (2010).

\subsection{Stellar parameters}

Stellar effective temperatures of the individual stars were obtained from their $(V-I)$ colors using the calibrations of Ramírez $\&$ Meléndez (2005). To this end, we transformed the SDSS $g, r, i$ magnitudes to the Johnson-Cousins system following the prescriptions of Jordi et al. (2006) and adopting a cluster reddening of $E(B-V)=0.08 \mathrm{mag}$ (Dotter et al. 2011). Although this is larger than the value listed by Harris (1996, 2010 edition), it is more in line with the reddening maps of Schlegel et al. (1998) and Schlafly et al. (2011), who list 0.05 and $0.06 \mathrm{mag}$, respectively, and it also gave the best representation of the SDSS CMD in Fig. 1. The typical uncertainty on $T_{\text {eff }}$ due to the (small) photometric errors in the SDSS and the calibrations is $\sim 50 \mathrm{~K}$.

Next, the surface gravities were obtained using the standard equations of stellar structure, where we used the above 
temperature, a stellar mass of $0.8 M_{\odot}$ for the RGB and $0.6 M_{\odot}$ for AGB stars, and a distance to Pal 5's center of $23.2 \mathrm{kpc}$. As an initial estimate of the stars' metallicity that enters the bolometric corrections, we adopted the photometric GC mean of -1.35 dex. One uncertain factor is the pronounced presence of mass segregation within Pal 5 and throughout its tails (Koch et al. 2004), which could also affect the analysis of our stars that are located throughout the cluster. Propagating the errors on all the above quantities translates into typical gravity uncertainties of 0.15 dex.

Similar to our previous work (Koch et al. 2009; Koch \& Côté 2010), we derived microturbulence velocities, $\xi$, using an empirical calibration of $\xi$ with $T_{\text {eff }}$ based on the halo stars of Roederer et al. (2014). This relation reads: 4.567-6.2694 $\times 10^{-4} \times T_{\text {eff }}$. The inferred uncertainty from the scatter around this relation is $0.10 \mathrm{~km} \mathrm{~s}^{-1}$.

While a global cluster metallicity is available for Pal 5 (e.g., Dotter et al. 2011) and previous low-resolution studies have measured metallicities of individual stars (e.g., Kuzma et al. 2015; see also Sect. 5.1), we opted to obtain initial metallicities of our targets that enter the stellar atmospheres from the Mg I line index. This index uses the strong $\mathrm{Mg}$ triplet lines at 5167 and $5173 \AA$ and is defined and calibrated on the scale of Carretta \& Gratton (1997) as in Walker et al. (2007) and Eq. (2) in Koch et al. (2009). For this, we assume a horizontal branch magnitude of $V_{\mathrm{HB}}=17.51 \mathrm{mag}$ (Harris 1996, 2010 edition). These values should only be taken as a general estimate for the metallicity distribution, since the $\mathrm{Mg}$ triplet has also dependencies on temperature and gravity. In particular, we note a trend of $[\mathrm{Fe} / \mathrm{H}]_{\mathrm{Mg}}$ with effective temperature. To identify possible causes, we consulted the Schlegel et al. (1998) maps and SDSS photometry for signs of differential reddening (following the approach of Milone et al. 2012; Kacharov et al. 2014). Either method indicates a very homogeneous reddening across the face of Pal 5 with a $1 \sigma$-scatter well below $0.01 \mathrm{mag}$. Similarly, the actual value of $E(B-V)$ only leads to a systematic shift of our $T_{\text {eff-scale, which has no bear- }}$ ing on the purported trend with metallicity. When accounting for uncertainties in our measurements, the correlation coefficient of metallicity with temperature becomes $0.25 \pm 0.35$ and we conclude that any possible trend is driven by statistical uncertainties.

All stellar parameters we have used in creating our stellar atmospheres are listed in Table 2 and plotted in Fig. 2.

\subsection{Co-added abundance measurements}

Consistent with our previous work, we have median-combined the spectra, irrespective of evolutionary status, after weighting by their SNR. This led to an SNR of $\sim 30$ per pixel at $6600 \AA$. Equivalent widths (EWs) were measured from the line lists of Koch et al. (2016) and Ruchti et al. (2016) by fitting Gaussian profiles with the splot task within the Image Reduction and Analysis Facility (IRAF). These line lists and the EW measurements are presented in Table 3.

In the following analysis, we have used the 2014 version of the stellar abundance code MOOG (Sneden 1973). From the stellar parameters derived in Sect. 4, we created individual stellar atmospheres for each star. Specifically, the ATLAS grid of Kurucz' one-dimensional 72-layer, plane-parallel, line-blanketed models without convective overshoot was interpolated assuming local thermodynamic equilibrium (LTE) for all species, together with the $\alpha$-enhanced opacity distribution functions, AODFNEW. We then computed theoretical EWs for all transitions using MOOG's ewfind driver and combined them into a mean $\langle E W\rangle$ by applying

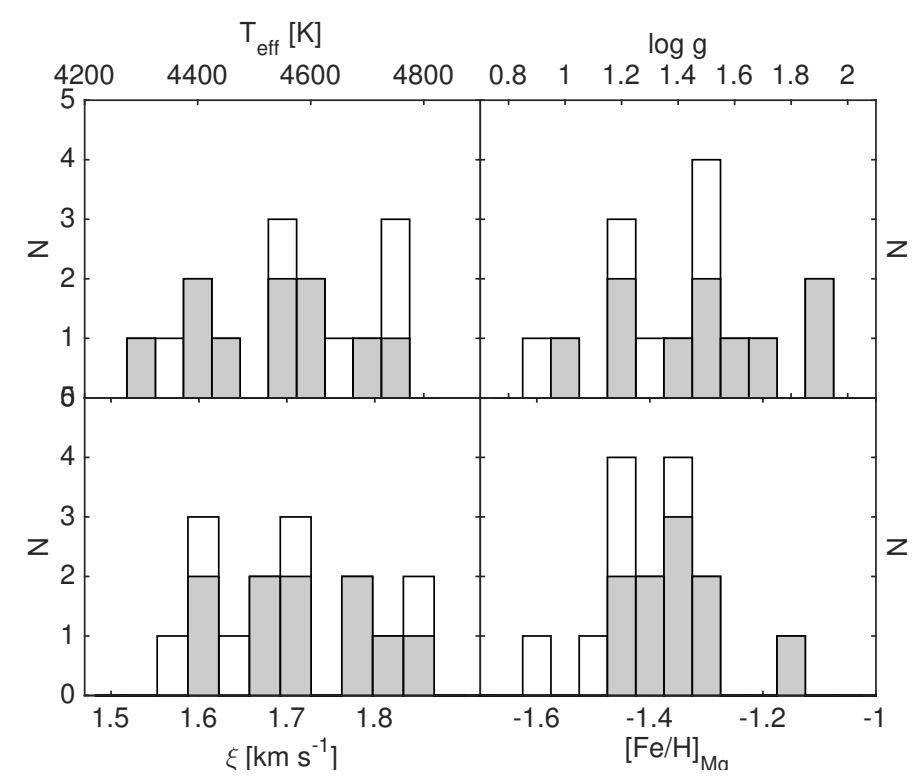

Fig. 2. Histogram of stellar parameters. Solid bars are for RGB stars and clear ones for the AGB subsample.

Table 3. Line list.

\begin{tabular}{|c|c|c|c|c|c|c|}
\hline \multirow{2}{*}{$\begin{array}{c}\lambda \\
{[\AA]}\end{array}$} & \multirow{2}{*}{ Species } & \multirow{2}{*}{$\begin{array}{l}\text { E.P. } \\
{[\mathrm{eV}]}\end{array}$} & \multirow{2}{*}{$\log g f$} & \multicolumn{3}{|c|}{$\langle E W\rangle[\mathrm{m} \AA]$} \\
\hline & & & & All & RGB & AGB \\
\hline 5682.633 & $\mathrm{NaI}$ & 2.102 & -0.700 & 61.3 & 68.9 & 35.9 \\
\hline 5688.205 & $\mathrm{NaI}$ & 2.104 & -0.404 & 91.9 & 96.0 & 38.4 \\
\hline 6154.225 & $\mathrm{NaI}$ & 2.102 & -1.547 & 11.8 & - & 10.2 \\
\hline 6160.747 & $\mathrm{NaI}$ & 2.104 & -1.246 & 31.0 & 35.8 & - \\
\hline 4571.096 & Mg I & 0.000 & -5.623 & 149.6 & 132.1 & 162.1 \\
\hline 4702.991 & $\mathrm{Mg}$ I & 4.346 & -0.440 & 171.5 & 162.7 & 157.2 \\
\hline 5711.088 & $\mathrm{Mg}$ I & 4.346 & -1.724 & 76.62 & 61.2 & 77.6 \\
\hline
\end{tabular}

Notes. Table 3 is available in its entirety in electronic form at the CDS.

the same SNR-based weights as for the observed spectra (see, e.g., Eq. (1). in Koch \& Côté 2010). Finally, the abundance ratio of each element was varied until the co-added $\langle E W\rangle$ matched the observed EW for each line to yield an integrated abundance ratio.

\subsection{Abundance errors}

We quantified the measurement error by standard procedures: firstly, the statistical error is given by the $1 \sigma$ line-to-line scatter and the number of lines, $N$, used to measure an element's abundance, each of which are listed in Table 4. For a few elements, only one weak feature was measurable and we varied the observed EW by $5 \mathrm{~m} \AA$ for those cases (which yielded reasonable fits to the line profile in splot). This led to typical uncertainties in the respective abundances of 0.15 dex.

Secondly, to determine the systematic uncertainties, we varied each of the stellar parameters by their typical uncertainty $\left(T_{\text {eff }} \pm 50 \mathrm{~K} ; \log g \pm 0.15 \mathrm{dex} ; \xi \pm 0.10 \mathrm{~km} \mathrm{~s}^{-1}\right)$ and re-ran the coaddition scheme. We note that this presupposes that all of the stars are affected by the same amount of error and the same sense of departure from the un-altered values. The difference for each element upon this variation in each parameter is listed in Table 4; there, we also indicate the effect of switching from 
Table 4. Abundance results from co-added spectra, where we also list results separately for the AGB- and RGB-only subsamples.

\begin{tabular}{|c|c|c|c|c|c|c|c|c|c|c|c|c|c|c|}
\hline \multirow{2}{*}{ Species } & {$[\mathrm{X} / \mathrm{Fe}]$} & $\sigma$ & $N$ & {$[X / \mathrm{Fe}]$} & $\sigma$ & $N$ & {$[X / \mathrm{Fe}]$} & $\sigma$ & $N$ & \multirow{2}{*}{$\begin{array}{l}\Delta T_{\mathrm{eff}} \\
\pm 50 \mathrm{~K}\end{array}$} & \multirow{2}{*}{$\begin{array}{c}\Delta \log g \\
\pm 0.15 \mathrm{dex}\end{array}$} & \multirow{2}{*}{$\begin{array}{c}\Delta \xi \\
\pm 0.1 \mathrm{~km} \mathrm{~s}^{-1}\end{array}$} & \multirow{2}{*}{ ODF } & \multirow{2}{*}{$\sigma_{\text {sys }}$} \\
\hline & \multicolumn{3}{|c|}{ All } & \multicolumn{3}{|c|}{ RGB } & \multicolumn{3}{|c|}{ AGB } & & & & & \\
\hline Fe I & -1.56 & 0.20 & 104 & -1.65 & 0.26 & 95 & -1.66 & 0.29 & 83 & \pm 0.05 & $<0.01$ & $\mp 0.03$ & $<0.01$ & 0.06 \\
\hline Fe II & -1.46 & 0.30 & 13 & -1.50 & 0.45 & 12 & -1.64 & 0.60 & 13 & $\mp 0.06$ & \pm 0.10 & $\mp 0.02$ & -0.05 & 0.13 \\
\hline $\mathrm{NaI}$ & 0.44 & 0.16 & 4 & 0.65 & 0.08 & 3 & 0.13 & 0.20 & 3 & $\mp 0.04$ & \pm 0.01 & \pm 0.01 & -0.01 & 0.04 \\
\hline $\operatorname{Mg} \mathrm{I}$ & 0.44 & 0.31 & 3 & 0.25 & 0.44 & 3 & 0.67 & 0.15 & 3 & $\mp 0.04$ & \pm 0.01 & \pm 0.02 & -0.02 & 0.05 \\
\hline Si I & 0.53 & 0.25 & 9 & 0.47 & 0.58 & 8 & 0.54 & 0.27 & 9 & $<0.01$ & $\mp 0.02$ & $<0.01$ & -0.01 & 0.02 \\
\hline $\mathrm{Ca} \mathrm{I}$ & 0.38 & 0.25 & 21 & 0.40 & 0.26 & 21 & 0.36 & 0.29 & 21 & $\mp 0.06$ & \pm 0.01 & \pm 0.05 & -0.04 & 0.09 \\
\hline Sc I & 0.25 & 0.07 & 2 & 0.38 & 0.23 & 2 & - & - & 0 & $\mp 0.09$ & \pm 0.01 & $<0.01$ & $<0.01$ & 0.09 \\
\hline Sc II & 0.09 & 0.13 & 6 & 0.16 & 0.10 & 6 & 0.28 & 0.31 & 6 & \pm 0.01 & $\mp 0.06$ & \pm 0.02 & -0.02 & 0.07 \\
\hline Ti I & 0.20 & 0.22 & 22 & 0.28 & 0.25 & 22 & 0.47 & 0.38 & 18 & $\mp 0.09$ & \pm 0.01 & \pm 0.03 & -0.03 & 0.10 \\
\hline Ti II & 0.22 & 0.28 & 10 & 0.28 & 0.41 & 10 & 0.44 & 0.42 & 10 & \pm 0.01 & $\mp 0.06$ & \pm 0.06 & -0.06 & 0.10 \\
\hline $\mathrm{V}_{\mathrm{I}}$ & -0.03 & 0.21 & 9 & 0.01 & 0.21 & 8 & 0.27 & 0.65 & 5 & $\mp 0.09$ & \pm 0.01 & \pm 0.01 & -0.01 & 0.09 \\
\hline Cr I & 0.00 & 0.33 & 5 & 0.10 & 0.41 & 4 & 0.12 & 0.49 & 5 & $\mp 0.09$ & \pm 0.01 & \pm 0.03 & -0.03 & 0.10 \\
\hline Cr II & 0.10 & - & 1 & 0.07 & - & 1 & - & - & 0 & \pm 0.03 & $\mp 0.06$ & \pm 0.02 & -0.02 & 0.07 \\
\hline Mn I & -0.10 & 0.24 & 8 & 0.07 & 0.28 & 8 & -0.13 & 0.34 & 8 & $\mp 0.06$ & \pm 0.01 & \pm 0.02 & -0.02 & 0.06 \\
\hline Co I & -0.10 & 0.07 & 3 & -0.01 & 0.08 & 3 & 0.18 & 0.08 & 2 & $\mp 0.05$ & $<0.01$ & \pm 0.01 & -0.01 & 0.05 \\
\hline $\mathrm{Ni} I$ & 0.05 & 0.24 & 18 & 0.12 & 0.17 & 17 & 0.11 & 0.46 & 15 & $\mp 0.03$ & $\mp 0.01$ & \pm 0.01 & -0.02 & 0.04 \\
\hline $\mathrm{Cu} \mathrm{I}$ & -0.49 & 0.22 & 2 & -0.21 & 0.01 & 2 & -0.18 & 0.15 & 2 & $\mp 0.06$ & $\mp 0.01$ & \pm 0.03 & -0.03 & 0.07 \\
\hline $\mathrm{Zn} \mathrm{I}$ & 0.05 & 0.05 & 2 & 0.04 & 0.38 & 2 & -0.10 & - & 1 & \pm 0.02 & $\mp 0.04$ & \pm 0.03 & -0.03 & 0.06 \\
\hline Y II & -0.35 & 0.05 & 2 & -0.32 & 0.03 & 2 & -0.35 & 0.02 & 2 & $\mp 0.02$ & $\mp 0.05$ & \pm 0.06 & -0.07 & 0.11 \\
\hline Zr II & -0.13 & 0.10 & 2 & - & - & 0 & - & - & - & $<0.01$ & $\mp 0.06$ & \pm 0.01 & -0.02 & 0.06 \\
\hline Ba II & 0.40 & 0.16 & 5 & 0.36 & 0.22 & 5 & 0.55 & 0.26 & 5 & $\mp 0.02$ & $\mp 0.04$ & \pm 0.06 & -0.09 & 0.12 \\
\hline La II & 0.13 & - & 1 & 0.29 & - & 1 & 0.26 & - & 1 & $\mp 0.01$ & $\mp 0.06$ & \pm 0.01 & -0.01 & 0.06 \\
\hline Nd II & 0.39 & - & 1 & 0.32 & - & 1 & - & - & - & $\mp 0.01$ & $\mp 0.06$ & \pm 0.02 & -0.02 & 0.07 \\
\hline Sm II & 0.24 & 0.10 & 4 & 0.41 & 0.22 & 4 & 0.32 & 0.09 & 2 & $\mp 0.01$ & $\mp 0.06$ & \pm 0.01 & -0.01 & 0.06 \\
\hline Eu II & 0.55 & - & 1 & - & - & 0 & 0.51 & - & 1 & $<0.01$ & $\mp 0.06$ & $<0.01$ & -0.01 & 0.06 \\
\hline
\end{tabular}

Notes. Abundance ratios for ionized species are given relative to Fe II. For iron itself, $[\mathrm{Fe} / \mathrm{H}]$ is listed. The line-to-line scatter $\sigma$ and number of measured lines, $N$, determine the statistical error, while the systematic uncertainties are indicated in the last five columns.

the $\alpha$-enhanced opacity distribution functions to the solar-scaled distributions, ODFNEW. Due to the strong correlations between temperature, gravity, and, as per our construction, the microturbulence, also the systematic uncertainties are not independent of each other. Thus, we caution that the total error we list in Table 4, which is merely the quadratic sum of all contributions, should be taken as a conservative upper limit at most.

In Koch \& Côté (2010), we investigated further error sources such as radial velocity uncertainties which can lead to additional line broadening, but found that this accounts for less than 0.04 dex in the error budget. Likewise, the accidental inclusion of foreground stars would add no more than 0.02 dex of abundance uncertainty.

\section{Abundance results}

The results from our co-added abundance measurements are presented in Table 4. All our abundances are on the scale of solar abundances from Asplund et al. (2009). Figures 3-5 show these abundance ratios in comparison with other Galactic components, such as halo field stars (from Roederer et al. 2014), the bulge (Johnson et al. 2012, 2014), and the disks (Koch \& Edvardsson 2002; Bensby et al. 2014). Furthermore we added GC data for M 5 as a representative of an undisturbed system at comparable metallicity (Ivans et al. 2001), the outer halo GCs Pal 3 and Pal 4 (Koch et al. 2009; Koch \& Côté 2010), and several objects that have been associated with the disrupting Sgr dwarf galaxy (see the caption of Fig. 3 for the color coding): Pal 12 (Cohen 2014); Terzan 7 (Sbordone et al. 2005); Arp 2 (Mottini et al. 2008); Terzan 8 and the massive M 54 (Carretta et al. 2014), and NGC 5053 and 5634 (Sbordone et al 2015). Finally, the tidally disturbed cluster NGC 5466 (Lamb et al. 2015) is shown as an orange hexagon.

\subsection{Iron abundance and metallicity}

The ensuing metallicity distribution from the Mg index (Fig. 2, bottom right) indicates a mean metallicity of $-1.38 \mathrm{dex}$. This is in excellent agreement with both photometric values of -1.4 dex (Dotter et al. 2011) and, to within their (large) uncertainties, the low-resolution mean values of Kuzma et al. (2015) and Ishigaki et al. (2016) of $-1.48 \pm 0.10$ and $-1.35 \pm 0.06$, respectively. All values can be reconciled if we split our sample into RGB stars only $(-1.33 \pm 0.09 \mathrm{dex})$ versus an AGB-only subset $(-1.47 \pm 0.08 \mathrm{dex})$. We note, however, that the nominal uncertainties on this index measurement are typically large so that conclusions drawn from the distribution function should be taken with caution compared to the more reliable iron abundance from individual lines. Smith et al. (2002) found a more metal-rich mean value, $-1.28 \pm 0.03 \mathrm{dex}$, from four high-SNR, medium resolution spectra, which is still consistent with the average $[\mathrm{Fe} / \mathrm{H}]$ from our sample when only considering RGB stars. 


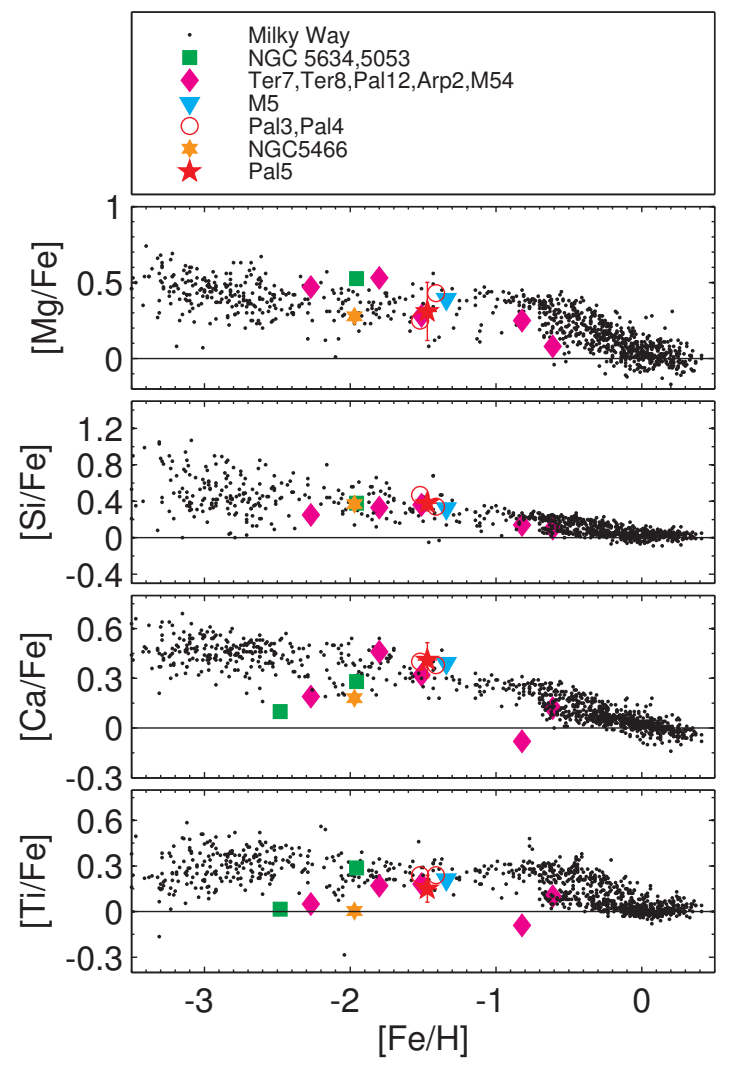

Fig. 3. Abundance results for the $\alpha$-elements. Literature data for the Milky Way (black dots) are from: halo - Roederer et al. (2014); bulge Johnson et al. (2012, 2014); disks - Bensby et al. (2014). Pal 5 is shown as a red star symbol, while other GCs are from Sbordone et al. (2015) NGC 5053, 5634 (green squares); Cohen (2014); Sbordone et al. (2007); Mottini et al. (2008); Carretta et al. (2014) for Pal 12, Ter 7, Arp 2, Ter 8, and M 54 (magenta diamonds). Furthermore, M 5 is shown as a cyan triangle (Ivans et al. 2001), the outer halo GCs Pal 3 and Pal 4 as open circles (Koch et al. 2009; Koch \& Côté 2010), and the disrupted NGC 5466 as an orange hexagon (Lamb et al. 2015).

From the co-added spectrum, we measured 104 Fe I and $13 \mathrm{Fe}$ II lines to arrive at an $\mathrm{Fe}$-abundance that is marginally lower than the aforementioned values, namely $[\mathrm{Fe} / \mathrm{H}]=-1.56 \pm 0.02 \pm 0.06 \mathrm{dex}$ (statistical and systematic errors, respectively) based on the neutral species. Ionization equilibrium is marginally reached in this co-added approach, at $[\mathrm{Fe} \mathrm{I} / \mathrm{Fe} \mathrm{II}]=-0.10 \pm 0.09 \mathrm{dex}$ (Table 4 ). In the following discussions, abundance ratios of ionized species will be referenced to the iron abundance from Fe II lines.

\subsection{Light elements: $\mathrm{Na}$}

Unfortunately, both of the strong $\mathrm{Na} \mathrm{D}$ lines fall on the gap between adjacent orders so we derived the Na-abundance from the weaker 5682, 5688, 6154, and $6160 \AA$ lines. No oxygen lines were detectable in the co-added spectrum. At 0.44 dex, the mean, co-added $[\mathrm{Na} / \mathrm{Fe}]$ abundance ratio of Pal 5 is high compared to halo stars at the same metallicity, which usually show solar to subsolar values. The corrections for Non-Local Thermodynamic Equilibrium (NLTE) in individual stars with stellar parameters as in our Pal 5 sample are on the order of -0.10 dex (Lind et al. 2011). Generally, the presence of strong Na lines could provide evidence of Na-strong, second generation GC stars amongst the sample. In fact, a pronounced light-element spread in Pal 5
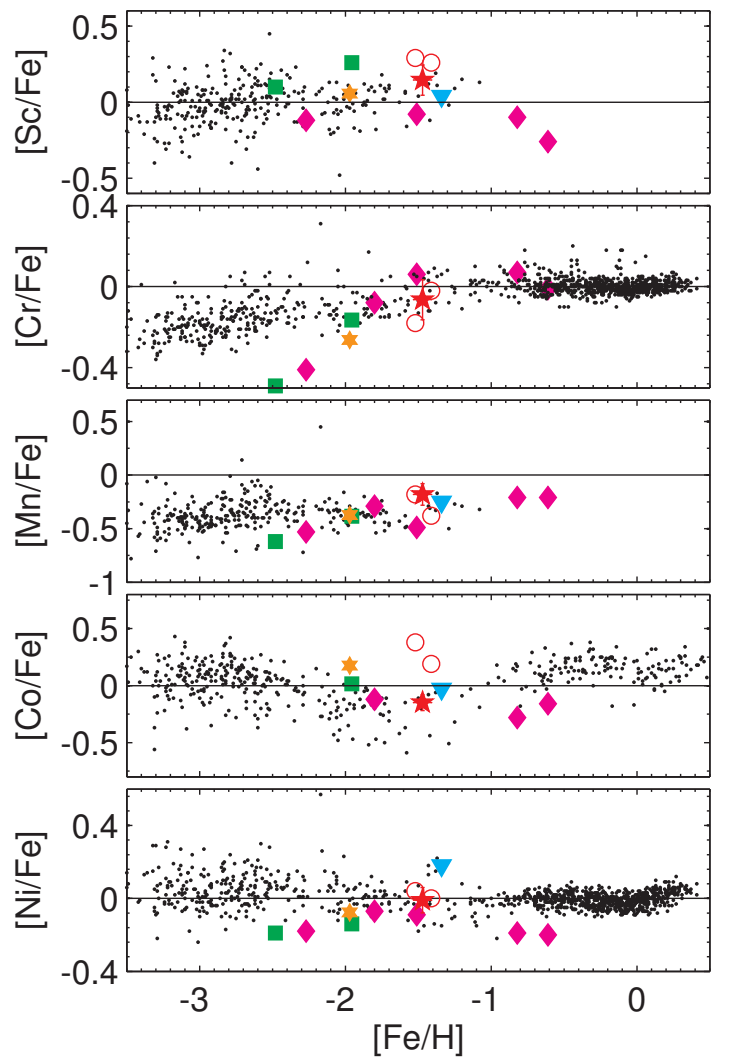

Fig. 4. Same as Fig. 3, but for Fe-peak and heavy elements.

of 0.3 dex (with a full range of 0.6 dex) was already noted by Smith et al. (2002). We further discuss the sensitivity of our analysis method to these light element variations in Sect. 6.

\subsection{Alpha-elements: $\mathrm{Mg}, \mathrm{Si}, \mathrm{Ca}, \mathrm{Ti}$}

Pal 5 shows an enhancement in the $\alpha$-elements, which overlaps with the halo distribution by merit of the latter's broad scatter. A straight average over all four elements, $\mathrm{Mg}, \mathrm{Si}, \mathrm{Ca}$, and $\mathrm{Ti}$, yields $[\alpha / \mathrm{Fe}]=0.39$ dex, although differences in each element's production channel renders other weighting schemes more appropriate. Smith et al. (2002) found the abundances of these elements to be significantly lower $(\langle[\mathrm{Si}, \mathrm{Ca}, \mathrm{Ti} / \mathrm{Fe}]\rangle=0.16$, while we find 0.37 from those three elements) when compared to halo field stars or the similarly metal-poor GC M 5 (Ivans et al. 2001; Koch \& McWilliam 2010). Our data indicate a depletion from the $\alpha$-plateau by $\sim 0.2$ dex, while the remainder of the elements, in particular $\mathrm{Mg}$, lie very close the canonical value of $\sim 0.4$ dex. This difference is curious - both $\mathrm{Mg}$ and Ti are $\alpha$-elements, but the $\mathrm{Mg}$-production is also affected by proton-capture reactions, leading to the conversion into $\mathrm{Al}$, while $\mathrm{Ti}$ is not affected by this channel. The lack of a significant $\mathrm{Mg}$-depletion could then indicate that proton-capture processing in the first generation polluters for this cluster was rather inefficient. Unfortunately, no Al could be measured owing to an SNR of $\sim 28$ in the region of the commonly used 6696, $6698 \AA$ A-lines. We note that both our measurement for $\mathrm{Mg}$ and $\mathrm{Ti}$ still overlap with the M 5-abundances (cyan triangles in Figs. 4-6) to within the errors. Our values are furthermore fully consistent with the measurements of individual stars in the M 54, which, at -1.5 has a very similar metallicity to Pal 5. This massive GC is thought to be the central cluster of the Sgr dwarf galaxy. Finally, we note that there is an excellent 


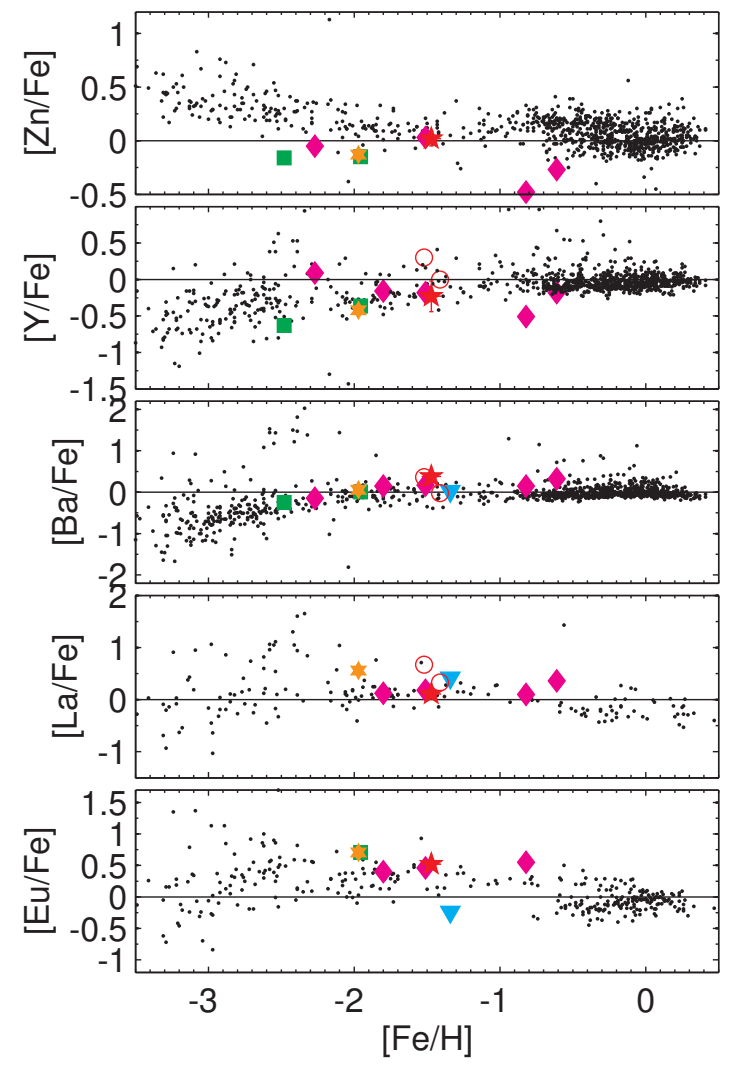

Fig. 5. Same as Fig. 3, but for neutron-capture elements. Eu disk abundances are from Koch \& Edvardsson (2002).

ionization balance between $\mathrm{Ti}$ I and II when taken relative to $\mathrm{Fe}$ I and Fe II.

\subsection{Fe-peak and heavy elements: Sc, V, Cr, Mn, Co, Ni, Cu, $\mathrm{Zn}$}

None of the Fe-peak elements show a surprise as they all fall in the regime of the halo field star and GC distributions (Fig. 4). We note that ionization equilibrium is not fulfilled for $\mathrm{Sc}$ and $\mathrm{Cr}$, but the measurements of their minority species are usually based on only one or two lines.

\subsection{Neutron-capture elements: $\mathrm{Zr}, \mathrm{Y}, \mathrm{Ba}, \mathrm{La}, \mathrm{Nd}, \mathrm{Sm}, \mathrm{Eu}$}

All of these elements have sound measurements, while $[\mathrm{Eu} / \mathrm{Fe}]$ is based on a marginal detection $(E W=17 \mathrm{~m} \AA)$ of the $6645 \AA$ line. As for the remainder of the elements, also the neutron-capture elements we determined (Fig. 5) are fully compatible with Galactic halo stars and GCs at similar metallicities. This indicates that Pal 5's early evolution was governed by the same enrichment processes as other, old and metal-poor GCs over a broad range of masses so that the subsequent strong mass-loss had no bearing on the chemical abundance patterns observed today.

\section{6. $R G B$ versus $A G B$ subsamples}

It has been known that AGB stars can have lower metal abundances when using photometric gravities in the abundance analysis, a fact that is commonly attributed to the impact of departures from the LTE assumption (Lapenna et al. 2015, and references therein). Thus we divided our samples into an AGB-only and a RGB-only sample and proceeded with EW measurements and abundance analyses in full analogy to the methods outlined above (see also Koch \& Côté 2010). These separate EW measurements and resulting abundance results are detailed in Tables 3 and 4. We note, however, the low SNR of the AGB sample and the values listed here should be used with caution.

The main effect is a lowering of the iron abundances as was already seen in the global metallicities (Sect. 5.1); upon this subdivision, however, an excellent ionization equilibrium for the AGB sample could be reached, which supports the suggestion that NLTE effects could be the driver of the abundance departures. A pronounced difference is seen in the $[\mathrm{Na} / \mathrm{Fe}]$ abundance ratios between AGB and RGB, with red giants having a markedly higher $\mathrm{Na}$ abundance by $0.5 \mathrm{dex}$. This may be due to different extents of the NLTE corrections in these types of stars. Furthermore, this is just what is expected if a Na-rich second generation of stars (which are also more He-rich than first generation stars) fails to reach or complete the AGB phase. We discuss this further in the context of light element variations within this $\mathrm{GC}$ in Sect. 7. The opposite is true for $\mathrm{Mg}$ : here, a low $\mathrm{Mg}$ in the RGB sample is contrasted by the AGBs' strong enhancement (Sect. 7; see also Mucciarelli et al. 2012). The remainder of the measured elements agree to with the error bars, which are inevitably larger due to the lower SNR of the subsamples.

\section{Comparison with other clusters}

The distinguishing feature of Pal 5 is clearly its severe state of tidal disruption. Nevertheless, all chemical abundance information had already been imprinted on its stars at an early stage before this dynamical alteration began. Thus, chemically speaking, it is a typical representative of the old, metal-poor halo population. This is highlighted in Figs. 3-5 through a comparison to a selection of other GCs that are interesting for a variety of reasons.

M5 is an inner halo $\left(R_{\mathrm{GC}}=6 \mathrm{kpc}\right) \mathrm{GC}$ with a metallicity similar to that of Pal 5. It is presently much more massive than the very low-mass Pal 5 (at $M_{V}=-8.81$ vs. $-5.17 \mathrm{mag}$ ) and shows only mild evidence of tidal distortion (Jordi \& Grebel 2010). While Smith et al. (2002) note a lower $[\alpha / \mathrm{Fe}]$ ratio in Pal 5 compared to M5, all other elements measured in that study and by us are fully compatible with those in M5.

In Figs. 3-5 we overplotted several objects that have been associated with Sagittarius. Given the complex star formation and enrichment history of this massive Galactic satellite, those GCs span a broad range of metallicities from $\sim-0.5$ to below -2 dex. In this regard, younger outer halo clusters such as Pal 12 $\left([\mathrm{Fe} /] \mathrm{H}=-0.8 \mathrm{dex} ; R_{\mathrm{GC}}=16 \mathrm{kpc}\right)$ show the depleted $[\alpha / \mathrm{Fe}] \mathrm{ra}-$ tios typical of environments with a low star formation efficiency. This value amounts to 0.06 dex for Pal 12 (Cohen 2014) and is significantly lower than found in Pal 5, despite the latter already having an enhancement lower than the halo average. Other such clusters, such as M 54 at the same metallicity as Pal 5, are very similar in most of the elements, again indicating that the dynamical history of these systems has not affected any of their basic chemical properties.

Located beyond $90 \mathrm{kpc}, \mathrm{Pal} 3$ and $\mathrm{Pal} 4$ are amongst the most distant GCs in the Milky Way halo. Despite their younger ages ( 10 Gyr compared to 12 Gyr for other, typical old GCs; MarínFranch et al. 2009; Dotter et al. 2011), the main conclusion from the co-added abundance analyses of Koch et al. (2009) and Koch \& Côté (2010) was that these outer halo clusters are chemically indistinguishable from the older ones in the inner halo and have thus experienced similar enrichment histories. Pal 3 and Pal 4 
have metallicities comparable to that of Pal 5 and most of their chemical abundances are very similar. In particular, Pal 3 has a lower mean $[\mathrm{Mg} / \mathrm{Fe}]$ ratio that is identical to the one we found for Pal 5. In a few cases, other element ratios deviate from the halo distribution and from Pal 5's abundances, such as higher $\mathrm{Sc}$ and enhanced Co for both clusters, and elevated $\mathrm{Zn}$ and $\mathrm{La}$ abundances in Pal 3; for the remaining elements, all the Pal's (3, 4 , and 5) element ratios agree within the uncertainties. Finally, we note that both of the studies mentioned above used the same technique of co-adding spectra and yielded very similar abundance results and precisions.

NGC 5466 is metal-poor $([\mathrm{Fe} / \mathrm{H}]=-1.97 \mathrm{dex})$ and shows tidal tails extending $\sim 4^{\circ}$ (Belokurov et al. 2006). An optical and infrared abundance analysis by Lamb et al. (2015) revealed typical GC abundance patterns for all studied elements in that this cluster shows light element variations and neutron-capture abundances indicative of pollution from AGB stars. Despite its state of disturbance, and a possible connection to the Sgr dwarf galaxy, Lamb et al. (2015) could not detect any obvious differences from the prevailing halo distribution at those low metallicities. NGC 5466's $\alpha$-elements are depleted to a similar extent as found for Pal 5, albeit at half a dex lower metallicity. We note that those authors used a combination of optical and infrared spectroscopy and accounted for departures from LTE for several elements, so that slight differences to the literature halo and GC samples (performed in LTE from optical data) occur naturally.

Another interesting reference object is the bulge cluster NGC 6712 at $[\mathrm{Fe} / \mathrm{H}]=-1.01 \mathrm{dex}$ (Yong et al. 2008). This object has lost a considerable amount of its mass as manifested in its mass and luminosity functions (de Marchi et al. 1999). It also shows $\mathrm{O}$ and $\mathrm{Na}$ abundance variations as strong as in any other GC, but which are not seen in halo field stars (e.g., Geisler et al. 2007). From the sheer large extent of the $\mathrm{O}$ and $\mathrm{Na}$ abundance variations in their sample of five stars (with full range of 0.6 dex and $1 \sigma$ scatter of 0.28 dex both in $\mathrm{Na}$ and $\mathrm{O}$ ), Yong et al. (2008) concluded that this GC must have been much more massive in the past in order to allow for these element variations to develop across the cluster's multiple populations. Such large spreads were also found by Smith et al. (2002) in Pal 5, which also has lost up to $90 \%$ of its initial mass. Therefore, it is timely to ask whether such light element variations could be detected in an abundance analysis based on co-added spectra.

\section{Light element variations}

The wavelength range of our spectra allowed us, in principle, to estimate the carbon-richness of the stars based on the $\mathrm{CH} G$-band at $\sim 4300 \AA$. The derived $\mathrm{CH}$-index provides a well-calibrated tool measured on low-resolution spectra, which often results in bimodal distributions indicative of the complex chemical enrichment processes in GCs (e.g., Smith 1985; Kayser et al. 2008). While we could attempt to measure a similar band-index, we note that our spectra cut-off redwards of the bluest end of the $\mathrm{CH}$-bandpass commonly used in such population studies (i.e., at $4298 \AA$ instead of the limit of $4285 \AA$; e.g., Eq. (2) in Kayser et al. 2008). This renders the zeropoint of our measurements incommensurable. It is, however, still very useful in identifying possible abundance spreads or outliers such as $\mathrm{CH}$-strong or -weak stars.

As Fig. 6 indicates, one object (the AGB-star J at 3.5' or 1.3 half-light radii) has a significantly lower $\mathrm{CH}$-index indicating that it is carbon-weak. The $\mathrm{CH}$ band strength is usually anticorrelated with the $\mathrm{N}$-abundance, which is commonly quantified by $\mathrm{CN}$-band index, in the sense that $\mathrm{CH}$-weak stars tend

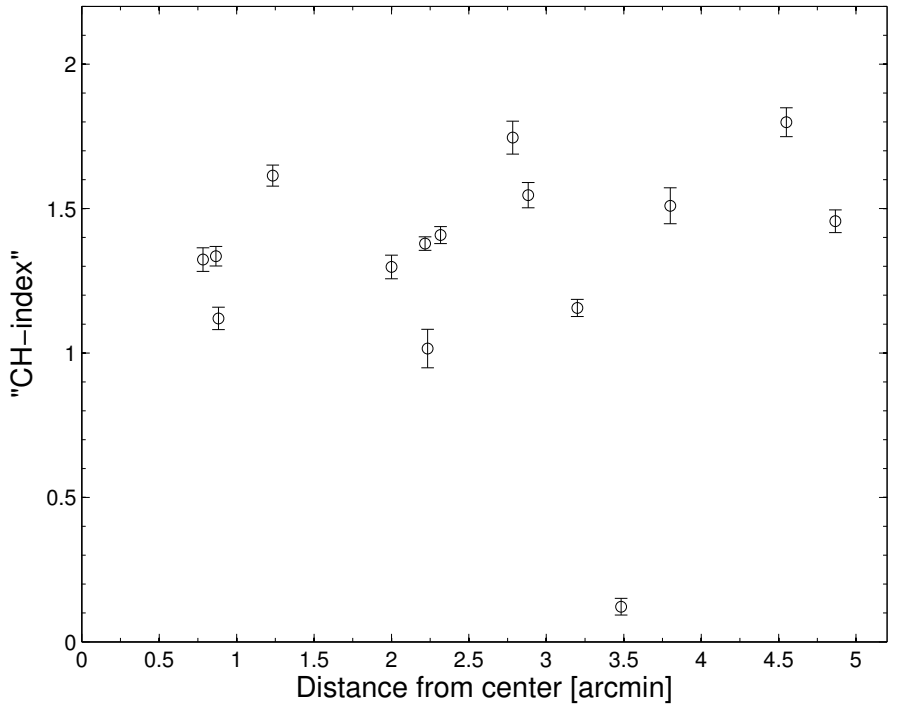

Fig. 6. Pseudo-CH-index versus position in the GC. The $\mathrm{CH}$-weak AGB-star Pal-J clearly stands out.

to be CN-strong (e.g., Kayser et al. 2008; Martell et al. 2010), so we should expect star $\mathbf{J}$ to also have a significantly stronger CN band. However, as already noted by Smith (1985), this is not the case and star $\mathrm{J}$ is also $\mathrm{CN}$-weak, which prompted him to invoke metallicity effects to explain the extraordinarily low $\mathrm{C}$-abundance. Indeed, star $\mathbf{J}$ is the member with the lowest metallicity in our sample.

Our method explicitly assumes that none of the chemical elements shows any abundance spread. Given the evidence of lightelement $(\mathrm{O}, \mathrm{Na}, \mathrm{Mg}, \mathrm{Al})$ variations in every $\mathrm{GC}$ studied to date, this is clearly a false premise. In order to test how our co-added abundance derivation responds to the presence of an abundance spread, we emulated a Na-O anti-correlation by randomly populating this abundance space with $10^{5}$ stars, following the dilution model for M 5 from Carretta et al. (2009). This GC is very similar to Pal 5 in most respects (Ivans et al. 2001; Carretta et al. 2009). The ratio of first generation (low-Na, high-O) to second generation stars (high-Na, low-O) was chosen as 30:70 (e.g., Carretta 2013). Out of this random sample, we drew 15 random values (corresponding to the number of targets) and predicted individual EWs for each with MOOG, ultimately co-adding them as before (Sect. 4.2).

As a result, we found no correlation of the combined EW with the sampling of Na-poor and Na-rich stars from the mock cluster, as quantified by the $[\mathrm{Na} / \mathrm{Fe}]$ interquartile range of the input sample. This means that co-added EW measurements or syntheses of integrated-light spectra (as also in, e.g., Koch \& Côté 2010; Sakari et al. 2013; Schönebeck et al. 2014) are rather insensitive to any spread in chemical elements, as exemplified here by sodium; even more so, since for Pal 5, a large Na-spread of 0.6 dex is already known from high-resolution measurements of four individual stars (Smith et al. 2002).

We note a trend in the $\mathrm{Na}$ and $\mathrm{Mg}$ abundances when performing an analysis on the AGB- and RGB-only subsamples, in that a systematically lower $\mathrm{Na}$ of the AGB sample goes along with a high Mg-abundance (Sect. 5.6). Conversely, the RGBsample appears depleted in $\mathrm{Mg}$ and enhanced in $\mathrm{Na}$. For want of a measurement of oxygen in either spectrum, we can take $\mathrm{Mg}$ as proxy for $\mathrm{O}$ as they are both produced via hydrostatic burning, albeit in different cycles that would render $\mathrm{Mg}-\mathrm{Al}$ a more suitable comparison. In this simplistic scenario, our measured $\mathrm{Mg}$ 
and $\mathrm{Na}$ abundances would reflect the presence of light element variations in analogy to the $\mathrm{Na}-\mathrm{O}$ anti-correlation, if we were to presume that the AGB spectrum was dominated by first generation (high $\mathrm{Mg}$, low $\mathrm{Na}$ ) stars. This is also in line with findings in some GCs that Na- and He-rich second generation stars can fail to reach or complete their AGB-phase (e.g., McLean et al. 2016).

\section{Summary and conclusions}

We determined chemical abundance ratios for various tracers of chemical evolution in the tidally disrupted GC Pal 5 using the co-addition technique we developed in earlier studies of remote Milky Way satellites. While this technique only allows us to measure the integrated properties of the stellar system, our results are fully compatible with results from a low number of individual high-resolution spectra (four stars of Smith et al. 2002). As a result, we found that Pal 5 is not unusual in any regard and that it follows the abundance trends of metal-poor GCs very closely, indicating that tidal perturbations over the course of Gyrs of evolution have no impact whatsoever on the chemical properties of these systems, although the present-day mass of GCs remains one of the main drivers of the extent of the observed light element variations (Carretta et al. 2010).

We were able to measure mean abundance ratios for 20 elements to high precision, however, our statistical tests have shown that the method of co-adding spectra is not sensitive to disentangling abundance variations such as the $\mathrm{Na}-\mathrm{O}$ anti-correlation, even if spreads of the typical, high degree of 0.6 dex are present. To better characterize those, individual spectra of a large number of stars are still the most viable course.

Acknowledgements. The authors thank Kyle Cudworth and Andrea Schweitze for making their membership catalogue available to us. We are grateful to the anonymous referee for a very fast and careful report. This work was based on observations obtained at the W. M. Keck Observatory, which is operated jointly by the California Institute of Technology and the University of California. We are grateful to the W. M. Keck Foundation for their vision and generosity. We recognize the great importance of Mauna Kea to both the native Hawaiian and astronomical communities, and we are grateful for the opportunity to observe from this special place.

\section{References}

Alam, S., Albareti, F. D., Allende Prieto, C., et al. 2015, ApJS, 219, 12 Asplund, M., Grevesse, N., Sauval, A. J., \& Scott, P. 2009, ARA\&A, 47, 481 Baumgardt, H., Côté, P., Hilker, M., et al. 2009, MNRAS, 396, 2051

Belokurov, V., Evans, N. W., Irwin, M. J., Hewett, P. C., \& Wilkinson, M. I. 2006, ApJ, 637, L29

Bensby, T., Feltzing, S., \& Oey, M. S. 2014, A\&A, 562, A71

Carretta, E. 2013, A\&A, 557, A128

Carretta, E., \& Gratton, R. G. 1997, A\&AS, 121, 95

Carretta, E., Bragaglia, A., Gratton, R. G., et al. 2009, A\&A, 505, 117

Carretta, E., Bragaglia, A., Gratton, R. G., et al. 2010, A\&A, 516, A55

Cayrel, R., Depagne, E., Spite, M., et al. 2004, A\&A, 416, 1117

Côté, P., Djorgovski, S. G., Meylan, G., Castro, S., \& McCarthy, J. K. 2002, ApJ, 574,783

Dehnen, W., Odenkirchen, M., Grebel, E. K., \& Rix, H.-W. 2004, AJ, 127, 2753

de Marchi, G., Leibundgut, B., Paresce, F., \& Pulone, L. 1999, A\&A, 343, L9

Dotter, A., Sarajedini, A., \& Anderson, J. 2011, ApJ, 738, 74
Frank, M. J., Hilker, M., Baumgardt, H., et al. 2012, MNRAS, 423, 2917 Geisler, D., Wallerstein, G., Smith, V. V., \& Casetti-Dinescu, D. I. 2007, PASP, 119,939

Gratton, R. G., Carretta, E., \& Bragaglia, A. 2012, A\&ARv, 20, 50

Hanke, M., Koch, A., Hansen, C. J., \& McWilliam, A. 2017, A\&A, 599, A97

Harris, W. E. 1996, AJ, 112, 1487

Ishigaki, M. N., Hwang, N., Chiba, M., \& Aoki, W. 2016, ApJ, 823, 157

Ivans, I. I., Kraft, R. P., Sneden, C., et al. 2001, AJ, 122, 1438

Johnson, C. I., Rich, R. M., Kobayashi, C., \& Fulbright, J. P. 2012, ApJ, 749, 175

Johnson, C. I., Rich, R. M., Kobayashi, C., Kunder, A., \& Koch, A. 2014, AJ, 148,67

Jordi, K., \& Grebel, E. K. 2010, A\&A, 522, A71

Jordi, K., Grebel, E. K., \& Ammon, K. 2006, A\&A, 460, 339

Jordi, K., Grebel, E. K., Hilker, M., et al. 2009, AJ, 137, 4586

Kacharov, N., Bianchini, P., Koch, A., et al. 2014, A\&A, 567, A69

Kayser, A., Hilker, M., Grebel, E. K., \& Willemsen, P. G. 2008, A\&A, 486, 437

Koch, A., \& Côté, P. 2010, A\&A, 517, A59

Koch, A., \& Edvardsson, B. 2002, A\&A, 381, 500

Koch, A., \& McWilliam, A. 2010, AJ, 139, 2289

Koch, A., \& McWilliam, A. 2014, A\&A, 565, A23

Koch, A., Grebel, E. K., Odenkirchen, M., Martínez-Delgado, D., \& Caldwell, J. A. R. 2004, AJ, 128, 2274

Koch, A., Côté, P., \& McWilliam, A. 2009, A\&A, 506, 729

Koch, A., McWilliam, A., Preston, G. W., \& Thompson, I. B. 2016, A\&A, 587, A124

Kuzma, P. B., Da Costa, G. S., Keller, S. C., \& Maunder, E. 2015, MNRAS, 446, 3297

Lamb, M. P., Venn, K. A., Shetrone, M. D., Sakari, C. M., \& Pritzl, B. J. 2015, MNRAS, 448, 42

Lapenna, E., Mucciarelli, A., Ferraro, F. R., et al. 2015, ApJ, 813, 97

Law, D. R., \& Majewski, S. R. 2010, ApJ, 718, 1128

Lind, K., Asplund, M., Barklem, P. S., \& Belyaev, A. K. 2011, A\&A, 528, A103

MacLean, B. T., Campbell, S. W., De Silva, G. M., et al. 2016, MNRAS, 460, L69

Marín-Franch, A., Aparicio, A., Piotto, G., et al. 2009, ApJ, 694, 1498

Marino, A. F., Sneden, C., Kraft, R. P., et al. 2011, A\&A, 532, A8

Marino, A. F., Milone, A. P., Karakas, A. I., et al. 2015, MNRAS, 450, 815

Martell, S. L., \& Grebel, E. K. 2010, A\&A, 519, A14

McWilliam, A., \& Bernstein, R. A. 2008, ApJ, 684, 326

Milone, A. P., Piotto, G., Bedin, L. R., et al. 2012, A\&A, 540, A16

Milone, A. P., Piotto, G., Renzini, A., et al. 2017, MNRAS, 464, 3636

Mucciarelli, A., Bellazzini, M., Ibata, R., et al. 2012, MNRAS, 426, 2889

Odenkirchen, M., Grebel, E. K., Dehnen, W., Rix, H.-W., \& Cudworth, K. M 2002, AJ, 124, 1497

Odenkirchen, M., Grebel, E. K., Dehnen, W., et al. 2003, AJ, 126, 2385

Odenkirchen, M., Grebel, E. K., Kayser, A., Rix, H.-W., \& Dehnen, W. 2009, AJ, 137, 3378

Pearson, S., Küpper, A. H. W., Johnston, K. V., \& Price-Whelan, A. M. 2015, ApJ, 799, 28

Piotto, G., Bedin, L. R., Anderson, J., et al. 2007, ApJ, 661, L53

Ramírez, I., \& Meléndez, J. 2005, ApJ, 626, 465

Roederer, I. U., Preston, G. W., Thompson, I. B., et al. 2014, AJ, 147, 136

Roederer, I. U., Mateo, M., Bailey, J. I., et al. 2016, MNRAS, 455, 2417

Ruchti, G. R., Feltzing, S., Lind, K., et al. 2016, MNRAS, 461, 2174

Sakari, C. M., Shetrone, M., Venn, K., McWilliam, A., \& Dotter, A. 2013, MNRAS, 434, 358

Sandage, A., \& Hartwick, F. D. A. 1977, AJ, 82, 459

Sbordone, L., Monaco, L., Moni Bidin, C., et al. 2015, A\&A, 579, A104

Schlafly, E. F., \& Finkbeiner, D. P. 2011, ApJ, 737, 103

Schlegel, D. J., Finkbeiner, D. P., \& Davis, M. 1998, ApJ, 500, 525

Schönebeck, F., Puzia, T. H., Pasquali, A., et al. 2014, A\&A, 572, A13

Schweitzer, A. E., Cudworth, K. M., \& Majewski, S. R. 1993, in The Globular Cluster-Galaxy Connection, ASP Conf. Ser., 48, 113

Smith, G. H. 1985, ApJ, 298, 249

Smith, G. H., Sneden, C., \& Kraft, R. P. 2002, AJ, 123, 1502

Tonry, J., \& Davis, M. 1979, AJ, 84, 1511

Vogt, S. S., Mateo, M., Olszewski, E. W., \& Keane, M. J. 1995, AJ, 109, 151

Walker, M. G., Mateo, M., Olszewski, E. W., et al. 2007, ApJS, 171, 389

Yong, D., Meléndez, J., Cunha, K., et al. 2008, ApJ, 689, 1020 